\title{
Variations saisonnières du comportement d'œstrus et de l'activité ovulatoire chez les brebis Chios et Serres en Grèce
}

\author{
M Avdi 1, MA Driancourt 2, P Chemineau 2* \\ 1 Université de Thessalonique, faculté d'agriculture, Thessalonique, Grèce \\ 2 INRA, physiologie reproduction, 37380 Nouzilly, France
}

(Reçu le 27 janvier 1992; accepté le 15 octobre 1992)

\begin{abstract}
Résumé - Vingt-sept brebis adultes de chacune des races Chios et Serres ont été maintenues hors reproduction pendant 2 années consécutives. L'œstrus est détecté 1 fois par jour et l'activité ovulatoire est appréciée par une analyse hebdomadaire de la progestérone circulante et par une endoscopie mensuelle des ovaires. Les brebis Chios ont des durées d'anœstrus et d'anovulation significativement plus courtes et plus variables que les brebis Serres: anœstrus: $109 \pm 8$ ( $m \pm e s m)$ vs $178 \pm 5$ j $(p<0,001)$; anovulation: $63 \pm 8$ vs $149 \pm 6$ jours $(p<0,001)$. Le pourcentage de brebis manifestant au moins un cestrus par mois ou au moins une ovulation par mois est significativement plus élevé de mars à juillet chez les brebis Chios que chez les brebis Serres. Cinq (première année) et 2 (deuxième année) brebis Chios conservent une activité ovulatoire pendant cette période alors que toutes les brebis Serres s'arrêtent. Le taux d'ovulation moyen est plus élevé chez les brebis Chios que chez les brebis Serres: $3,25 \pm 0,15$ vs $1,45 \pm 0,04(p<0,001)$. La durée du comportement d'œstrus est plus longue chez les brebis Chios que chez les brebis Serres: $1,8 \pm 0,05$ vs 1,5 $\pm 0,06 j$ $(p=0,002)$. Chez les brebis Chios, la durée de la première période anovulatoire est corrélée significativement avec celle de la seconde $(r=0,43)$; dans la race Serres le taux d'ovulation est positivement corrélé avec le début de la saison sexuelle. Les données présentées suggèrent que (1) d'une part, le printemps n'est pas la période optimale pour la lutte, puisqu'à cette saison le pourcentage de brebis en cestrus et le taux d'ovulation sont réduits, (2) d'autre part, pour la race Chios, compte tenu de la forte variabilité dans le saisonnement de l'activité sexuelle et de la bonne corrélation entre années des caractéristiques de cette aptitude, la sélection contre ce caractère pourrait être intéressante dans cette race.
\end{abstract}

cestrus / ovulation / brebis / saison / race

Summary - Seasonal variation in oestrous behaviour and ovulatory activity in Chios and Serres ewes in Greece. Sheep are seasonal breeders, but a lower seasonality has been reported for sheep breeds from the Mediterranean countries enabling spring matings. To further substantiate this, the present study compared seasonal variation in oestrus behaviour and ovulation in two Greek breeds (Chios and Serres) maintained in the same environment. In 27 adult ewes of each breed, oestrus behaviour, the occurrence of ovulation and ovulation rate were monitored during 2 successive years by daily inspection by rams, weekly progesterone assay in circulating blood plasma and

* Correspondance et tirés à part 
monthly ovarian laparoscopy. The periods of anoestrus (109 \pm 8 (wk) vs $178 \pm 5 d$ ) and anovulation $(63 \pm 8$ vs $149 \pm 6 d$ ) were significantly shorter $(P<0.001$ and $P<0.001)$ and more variable in Chios than in Serres ewes. Furthermore, the percentage of ewes exhibiting oestrus at least once monthly was significantly higher from March to July in Chios than in Serres ewes. Continuous ovulation throughout the year was noted in 5 and 2 Chios ewes during year 1 and 2, respectively. Ovulation rate ( $3.25 \pm 0.15$ vs $1.45 \pm 0.04$ ) was higher in Chios than in Serres ewes $(P<0.001)$ while duration of oestrus was also longer in Chios ewes ( $1.8 \pm 0.05$ vs $1.5 \pm 0.06 \mathrm{~d} ; \mathrm{P}=0.002$ ). There were significant correlations between the duration of anovulatory periods of the 2 successive years in Chios $(r=$ 0.43 ) but not Serres ewes. Ovulation rate was positively related to the onset of the sexual season in Serres but not in Chios ewes. The present data suggest that i) the efficiency of spring mating in these breeds is suboptimal since it is a period when the percentage of ewes exhibiting oestrus and ovulation as well as ovulation rate are limited, ii) owing to the large variation in seasonality within Chios ewes and to the good between year correlation in features of seasonality, selection against seasonality in Chios ewes could be worthwhile.

estrus / ovulation / ewe / season / breed

\section{INTRODUCTION}

Sous les latitudes moyennes et élevées $\left(>40^{\circ}\right)$ la plupart des races ovines manifestent un saisonnement marqué de leur activité sexuelle. L'activité de comportement d'oestrus et l'activité ovulatoire s'arrêtent quelques semaines après le solstice d'hiver pour redémarrer peu de temps après celui d'été (Hafez, 1952). Ces variations provoquent une synchronisation étroite des naissances dans les troupeaux. Dans les zones tropicales, en revanche, des oestrus et des ovulations se produisent toute l'année sans qu'il soit possible de déterminer avec précision une saison d'anoestrus et une saison d'ovulation (Mahieu et al, 1989).

Entre ces 2 grandes zones géographiques, les régions subtropicales chaudes possèdent des races ovines dont le saisonnement de la reproduction est moins marqué. Dans le Bassin Méditerranéen la plupart des brebis sont fécondées au printemps (Khaldi, 1984; Perret, 1986; Lysandrides, 1987), les autres ne sont fécondées qu'à l'automne. De plus, l'aptitude des femelles à être fécondées au printemps présente une variabilité importante.
Dans le travail rapporté ici, les variations saisonnières du comportement d'œstrus et de l'activité ovulatoire ont été étudiées pendant 2 années consécutives chez 2 races ovines grecques élevées sous la même latitude mais connues pour présenter des caractéristiques de reproduction très différentes : la race Chios et la race Serres.

\section{MATÉRIELS ET MÉTHODES}

\section{Animaux et conditions d'élevage}

L'expérience a été réalisée à la station expérimentale de Halkidiki près de Thessalonique (latitude $40^{\circ} 15$ Nord), elle a duré pendant 24 mois consécutifs, de septembre à août (22 mois de détection d'cestrus et 24 mois de détection des ovulations). Vingt-sept femelles représentatives de chacune des 2 races Chios et Serres, agées d'au moins 2 ans en début d'expérimentation ont été choisies. Elles avaient toutes des antécédents normaux de reproduction et avaient mis bas au moins une fois. Elles ont reçu une alimentation constante au cours de l'expérimentation, constituée de foin à volonté, de maïs (600 g), d'orge $(400 \mathrm{~g})$, de pulpe de betterave déshydratée $(500 \mathrm{~g})$, de tourteau de coton $(240 \mathrm{~g})$ et de soja $(100 \mathrm{~g})$. Le poids vif des brebis Chios a 
diminué entre le début et la fin de l'expérience ( $m \pm$ esm; 73,4 $\pm 1,6$ vs $64,8 \pm 1,3 \mathrm{~kg} ; P<$ 0,001 ), alors que celui des brebis Serres a légèrement augmenté $(49,0 \pm 1,4$ vs $51,5 \pm 1,5 ; P<$ $0,01)$.

Les brebis 2 races ont été élevées séparément et laissées en contact visuel et tactile avec des béliers entiers de race Chios qui servent à la détection des chaleurs. Les femelles ont été maintenues hors reproduction pendant toute la durée de l'expérience.

\section{Détection de l'œstrus et mesure de l'activité ovulatoire}

Le comportement d'œstrus a été détecté une fois par jour (le matin entre 8 et $11 \mathrm{~h}$ ) avec des béliers Chios entiers munis de tabliers, pendant 22 mois (octobre à juillet). Le choix de béliers de race Chios a été fait, en raison de leur bonne libido. L'immobilisation de la femelle au chevauchement par le mâle était considérée comme caractéristique du comportement d'œstrus (Mauléon et Dauzier 1965).

L'activité ovulatoire a été apprécièe pendant 24 mois (septembre à août) par détermination hebdomadaire de la progestérone plasmatique sanguine (Terqui et Thimonier, 1974). Cette fréquence de prélèvements permet d'estimer les dates de première et dernière ovulations de la saison (Thimonier, 1978). Une endoscopie a été réalisée mensuellement sur chaque femelle lors du premier cycle du mois considéré, de 3-8 j après l'œstrus ou en fin de mois pour les femelles n'ayant pas manifesté de comportement d'œstrus (Thimonier et Mauléon, 1969). Aucune adhérence n'est observée en fin d'expérience, après abattage et examen post-mortem des tractus génitaux.

\section{Définitions et analyse des résultats}

La durée moyenne du comportement d'œstrus est le nombre de jours consécutifs pendant lesquels les femelles acceptent le chevauchement par le mâle au cours de la même période œstrale. La durée moyenne de l'activité d'œstrus ou d'ovulation est le nombre de jours qui séparent le premier du dernier oestrus ou la première de la dernière ovulation de la même saison sexuelle. La durée moyenne de l'anœstrus ou de l'anovulation est le nombre de j séparant le dernier œstrus ou la dernière ovulation de la saison sexuelle du premier cestrus ou de la première ovulation de la saison sexuelle suivante.

Des tests $F$ ont été utilisés pour comparer les variances, des tests $t$ appariés ou non pour comparer les moyennes et des tests $\chi^{2}$ pour comparer les proportions. Les effets de la race et du temps sont testés en analyse de variance avec facteurs répétés (Dagnélie, 1970).

\section{RÉSULTATS}

\section{Saisonnement de l'activité de comportement d'cestrus et de l'ovulation}

Dans les 2 races, il existe des variations saisonnières de l'activité sexuelle. Des périodes d'inactivité (Serres) ou de moindre activité (Chios) alternent avec des périodes pendant lesquelles les femelles manifestent un comportement d'œstrus et ovulent régulièrement (fig 1). Les dates de début et de fin de saison sont rapportées dans le tableau I. Pour tous les paramètres étudiés (dates de premier et dernier œstrus, dates de première et dernière ovulation), un effet de la race et un effet de l'année ( $n^{\circ}$ de saison sexuelle) sont mis en évidence par Anova $(P<0.001)$. Les brebis Serres terminent leur saison environ un mois plus tôt et la reprennent environ un mois plus tard que les brebis Chios. Les variabilités dans la date de début et de fin de la deuxième saison sexuelle sont significativement plus élevées chez les brebis Chios que chez les brebis Serres (tableau I).

Les durées d'ancestrus et d'anovulation sont significativement plus courtes (38 et $58 \%$ de moins) et plus variables dans la race Chios que dans la race Serres (tableau II). La durée de la saison est signifi- 

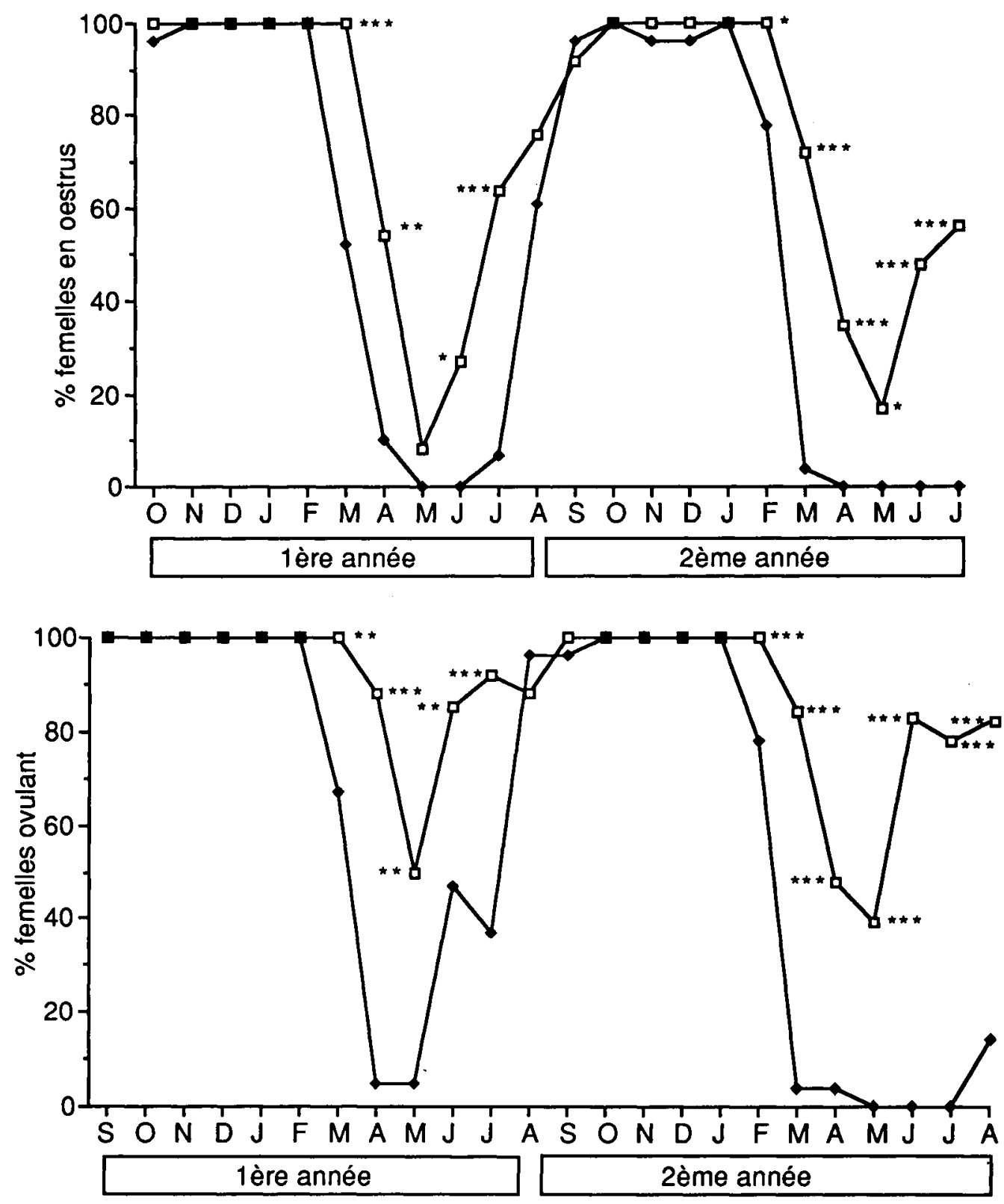

Fig 1. Variations saisonnières du pourcentage de femelles manifestant au moins un comportement d'cestrus par mois (en haut) et au moins une ovulation par mois (en bas), chez des brebis Chios ( $\square$ ) et Serres $(\downarrow)$ maintenues hors reproduction. Les étoiles indiquent une différence significative entre les proportions pour le mois considéré ${ }^{*} P<0,05 ;{ }^{* \star} P<0,01 ;{ }^{* \star *} P<0,001$ ). 
Table I. Date du début et de la fin de la saison sexuelle (premier et dernier oestrus, première et dernière ovulations) chez les brebis Chios et Serres.

Première saison sexuelle Deuxième saison sexuelle Troisième saison sexuelle

\begin{tabular}{|c|c|c|c|c|}
\hline & fin & début & fin & début \\
\hline $\begin{array}{l}\text { Brebis Chios } \\
\text { œstrus } \\
\text { ovulation }\end{array}$ & $\begin{array}{r}6 / 4 \pm 18 \\
12 / 4 \pm 16\end{array}$ & $\begin{array}{l}24 / 7 \pm 36 \\
30 / 6 \pm 24\end{array}$ & $\begin{array}{l}16 / 3 \pm 22 \\
18 / 3 \pm 26\end{array}$ & $\begin{array}{l}24 / 6 \pm 13 \\
21 / 6 \pm 29\end{array}$ \\
\hline $\begin{array}{c}\text { Brebis Serre } \\
\text { œstrus } \\
\text { ovulation }\end{array}$ & $\begin{array}{l}3 / 3^{* * *} \pm 18 \\
8 / 3^{* * *} \pm 15\end{array}$ & $\begin{array}{c}25 / 8^{* * * *} \pm 18^{* * *} \\
6 / 8^{\star * *} \pm 20\end{array}$ & $\begin{array}{l}6 / 2^{* * *} \pm 15^{*} \\
9 / 2^{* * *} \pm 11^{\star * *}\end{array}$ & $\underset{11 / 9^{\star \star \star}}{\text { ND }} \pm 5^{* \star \star}$ \\
\hline
\end{tabular}

Les résultats sont exprimés par les dates moyennes et les écarttypes (en jours); ND = non disponible; différences significatives entre races: : $=P<0,05 ; \cdots *=P<0,001$.

Table ll. Durée de la durée des saisons d'anœestrus et d'anovulation et des saisons d'œstrus et d'ovulation chez les brebis Chios et Serres.

$\frac{\text { Première contre saison }}{\text { Ancest } 1 \quad \text { Anov } 1} \frac{}{\text { Ancest } 2 \text { Anov } 2} \stackrel{\text { CEstrus Ovième contre saison }}{n}$

\begin{tabular}{|c|c|c|c|c|c|c|}
\hline $\begin{array}{l}\text { Brebis Chios } \\
m \pm \text { écart type (j) }\end{array}$ & $109 \pm 41$ & $63 \pm 42$ & $99 \pm 28$ & $85 \pm 50$ & $237 \pm 51$ & $281 \pm 52$ \\
\hline $\begin{array}{l}\text { Brebis Serres } \\
m \pm \text { écart type (j) }\end{array}$ & $176^{* \star *} \pm 26^{*}$ & $149^{\star \star *} \pm 30^{\star}$ & ND & $213^{* * *} \pm 11^{\star \star \star *}$ & $168^{* * *} \pm 21^{\star * * *}$ & $191^{* * *} \pm 24^{\star * * *}$ \\
\hline
\end{tabular}

Les résultats sont exprimées par les dates moyennes et les écarts types (en j); ND = hon disponible; différences significatives entre races: $*=P<0,05 ; * * *=P<0,001$.

cativement plus longue et plus variable chez la Chios que chez la Serres (59 et $42 \%$ de plus selon le critère retenu, œstrus et ovulation).

Exprimé en pourcentage de femelles manifestant au moins un œstrus ou une période d'ovulation par mois, des différences significatives apparaissent entre races (fig 1). Dans la race Serres, l'activité sexuelle est très fortement inhibée entre avril et juillet, alors que cet effet n'est visible qu'au seul mois de mai chez les brebis Chios, où le pourcentage de brebis ovulant n'est toutefois pas inférieur à $40 \%$. Individuellement, 5 brebis Chios ne manifestent aucun arrêt de leur activité ovula- 
toire pendant la période d'anœstrus, mais toutes arrêtent leur comportement d'œstrus pendant au moins un mois. Au cours de la seconde saison d'anœstrus, 2 d'entre elles continuent toujours à ovuler, les 3 autres s'arrêtent 28,52 et 73 j respectivement. Dans les 2 races étudiées, des ovulations silencieuses (ovulations sans comportement d'œstrus) sont observées; elles se manifestent de façon importante pendant la saison de faible activité sexuelle (avril à juillet).

\section{Durée du comportement d'cestrus et taux d'ovulation}

Le comportement d'œstrus dure significativement plus longtemps, en moyenne, chez la brebis Chios que chez la brebis Serres : 1,8 $\pm 0,05$ (sem) vs 1,5 j $\pm 0,06$, respectivement $(P=0,002)$. Une variation saisonnière significative est mise en évidence $(P=0,02)$ avec une plus longue durée d'œstrus en automne/hiver (fig 2). Le taux d'ovulation est significativement plus élevé chez les brebis Chios que chez les brebis Serres : $3,25 \pm 0,15$ vs $1,45 \pm$ 0,04 , respectivement $(P<0,001)$. Une va- riation saisonnière significative existe dans les 2 races $(P<0,001)$ ainsi qu'une interaction race $\mathrm{x}$ saison $(P=0,03)$ indiquant une plus forte amplitude chez les Chios que chez les Serres: les plus faibles taux d'ovulation sont observés au printemps et les plus élevés en automne (fig 3). La répétabilité du taux d'ovulation est de 0,33 chez les brebis Chios et de 0,07 chez les brebis Serres.

\section{Corrélations entre les différentes caractéristiques de l'activité sexuelle (tableau III)}

Dans la race Chios, les durées d'inactivité ou d'activité sont corrélées significativement entre elles et avec les dates de fin ou de début de saison sexuelle. La durée de la première période d'anovulation est significativement corrélée avec celle de la seconde $(+0,43)$. Aucune corrélation significative n'est mise en évidence entre dates ou durées de saison sexuelle et durée du comportement d'œstrus ou taux d'ovulation. Dans la race Serres, des corrélations significativement différentes de 0 entre dates et durées sont aussi observées,

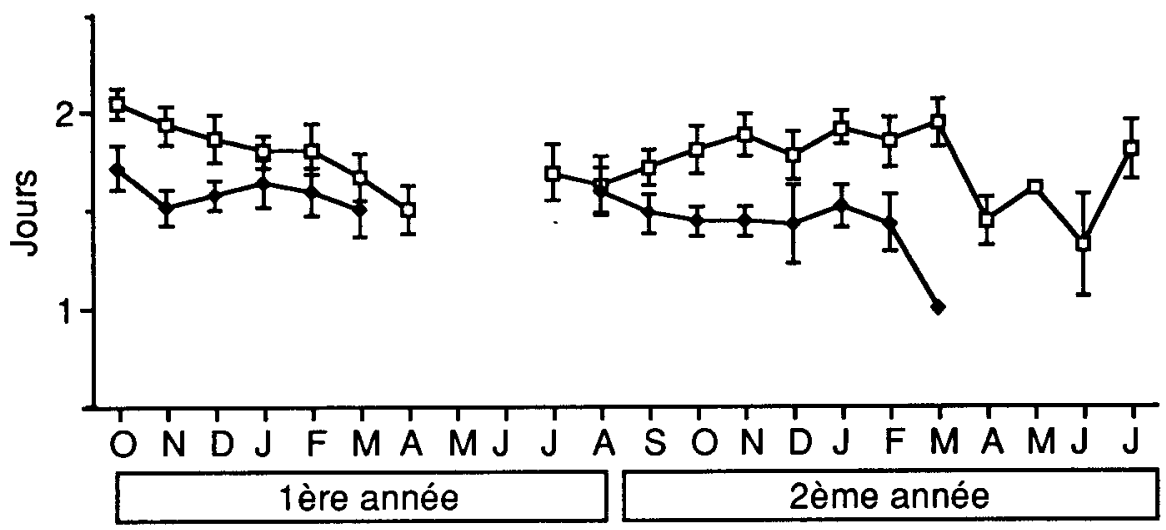

Fig 2. Variations saisonnières de la durée moyenne ( \pm sem) du comportement d'œstrus chez des brebis Chios $(\square)$ et Serres $(\bullet)$ maintenues hors reproduction. 


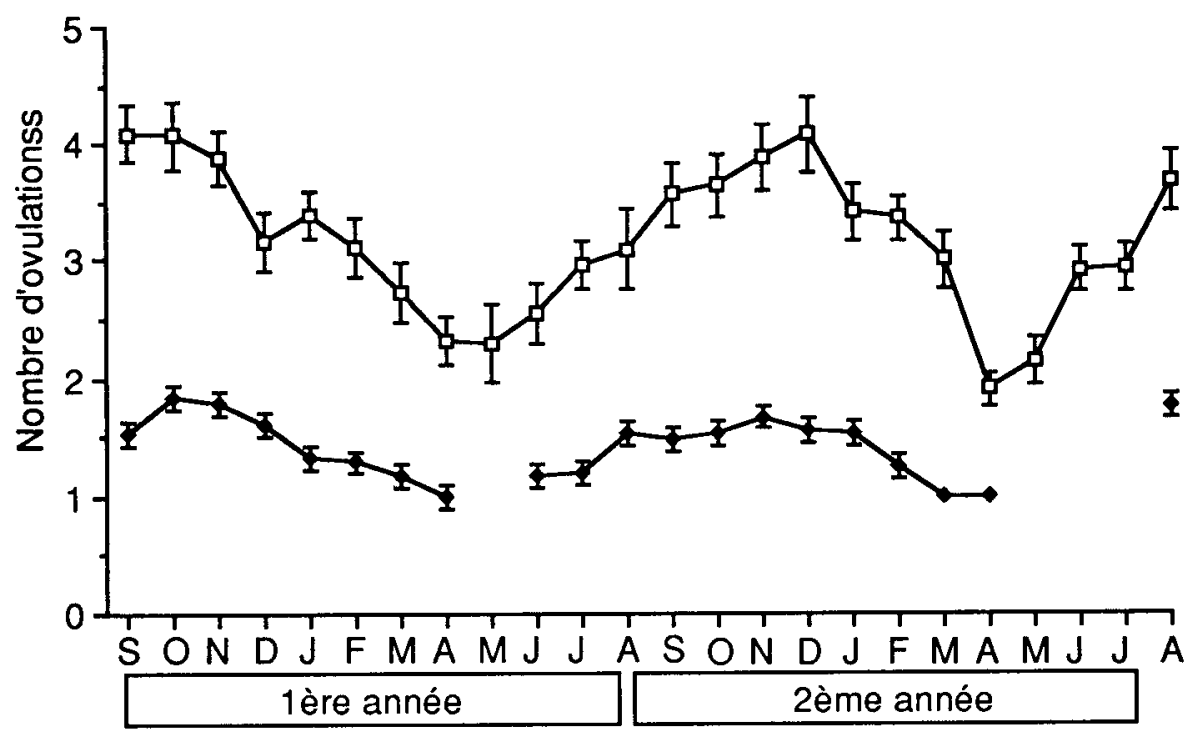

Fig 3. Variations saisonnières du nombre moyen d'ovulation ( \pm sem) chez des brebis Chios $(\square)$ et Serres $(\bullet)$ maintenues hors reproduction.

mais les durées d'anovulations successives ne sont pas significativement corrélées entre elles. Le taux d'ovulation est corrélé significativement avec la date de début d'œstrus $(+0,49)$, la date de début des ovulations $(+0,67)$ et avec la durée de la saison d'ovulation $(-0,53)$.

\section{DISCUSSION}

Les 2 races étudiées manifestent des caractéristiques très différentes d'activité sexuelle. La brebis Chios est fortement ovulante et relativement peu saisonnée, tandis que la brebis Serres est à faible taux d'ovulation et possède un saisonnement relativement important. Ces différences étaient attendues puisque ces 2 populations avaient été choisies en connaissant les performances moyennes de reproduction de ces 2 races (Hatjiminaoglou et al, 1985; Vosniakou et al, 1989). Il est intéressant toutefois de remarquer que les berceaux d'origine de cellesci ne sont éloignés que de quelques centaines de km : montagnes de la Grèce du Nord pour la Serres et lle de Chios pour la Chios. De plus, les animaux expérimentaux étudiés ici ont été élevés dans le même lieu et strictement dans les mêmes conditions d'entretien. Les différences mises en évidence semblent donc bien être d'origine génétique plutôt qu'environnementale.

Intra-race, la variabilité de la durée de la saison sexuelle est beaucoup plus importante chez les brebis Chios que chez les brebis Serres. Un cinquième des brebis Chios ne manifestent pas d'arrêt de leur activité ovulatoire pendant la première période d'anœstrus étudiée, alors que 


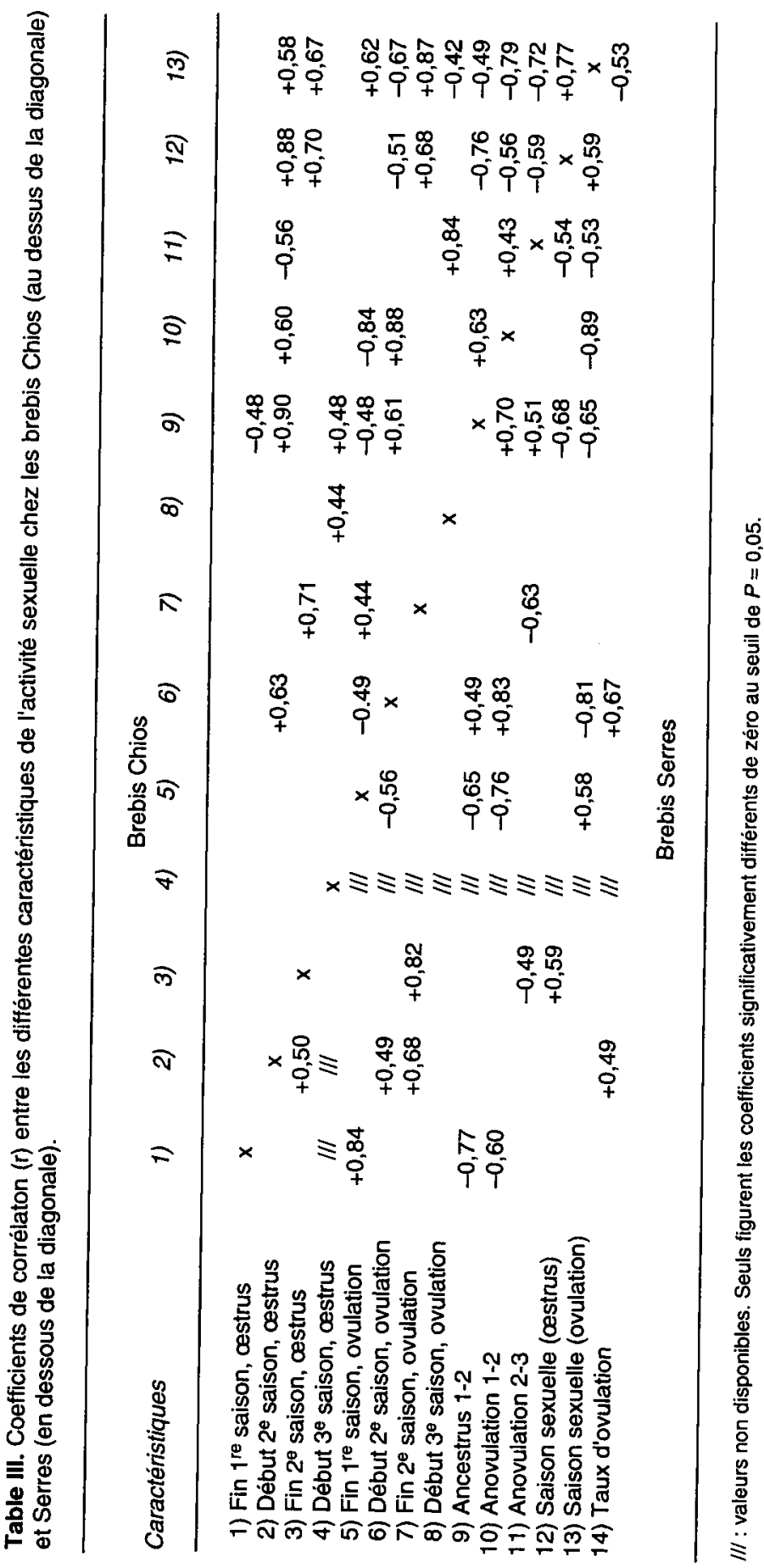


d'autres brebis s'arrêtent d'ovuler pendant plus de $150 \mathrm{j}$. II semble donc exister des différences individuelles importantes dans les caractéristiques du saisonnement pour cette population de brebis. Les fortes différences de variabilité de la durée de l'anovulation entre les brebis des 2 races confirment que les différences individuelles pour ce paramètre sont plus élevées chez les Chios que chez les Serres.

La comparaison des caractéristiques de l'activité sexuelle de ces 2 races décrites dans la présente expérience avec celles rapportées par Vosniakou et al, (1989) montre l'existence de différences marquées surtout dans la race Serres. Celle-ci apparaît en effet, plus saisonnée dans notre étude : 191 j de saison sexuelle (ovulation) contre $248 \mathrm{j}$ (Vosniakou et al, 1989). La définition du début de saison qui n'est pas identique dans les 2 expériences, pourraît expliquer une partie des différences observées. Malgré tout, dans les 2 cas les brebis Chios se montrent beaucoup moins saisonnées que les brebis Serres. Dans les 2 races, il existe un saisonnement plus marqué de l'apparition de l'œestrus que des ovulations, avec une fréquence plus élevée d'ovulations silencieuses pendant la saison de faible activité sexuelle. Une telle observation a déjà été rapportée dans d'autres races (Thimonier et Mauléon, 1969).

L'existence, chez les Chios d'une corrélation significative entre les durées montre que la durée de la période d'anovulation est un phénomène répétable d'une année sur l'autre. Par contre, la répétabilité de cette caractéristique est très faible chez les brebis Serres dont les ovulations apparaissent beaucoup plus tardivement la troisième année d'expérience. Peu d'auteurs ont, à notre connaissance, déterminé la répétabilité des caractéristiques de l'activité sexuelle saisonnière chez les petits ruminants. Une telle répétabilité (avec des valeurs très proches) a déjà été décrite chez la brebis Romanov (Thimonier, 1989) et chez la chèvre Alpine (Chemineau et al, 1992). Des répétabilités significativement différentes de 0 , mais de niveau moins élevé ont aussi été rapportées par Quirke et al (1988) chez des brebis de différentes races (Suffolk, Dorset, Rambouillet, Finnoises et $F_{1}$ ). La durée de la période de repos sexuel qui présente une forte variabilité, alliée à une bonne répétabilité, chez la brebis Chios, pourrait être une caractéristique intéressante à sélectionner dans cette race. Le fort taux d'ovulation manifesté par les brebis Chios dans cette étude confirme les résultats rapportés précédemment (Alifakiotis, 1986) et classe cette race parmi les plus ovulantes telles que la Finnoise (Young et al, 1985), la Romanov (Cornu et Cognié, 1985), la Mérinos Boroola (Bindon, 1984) ou la D'Man (LahlouKassi et Marie, 1985). Toutefois la faible répétabilité du taux d'ovulation suggère, au contraire de ce qui est observé chez la brebis Mérinos Boroola (Bindon, 1984), que le fort taux d'ovulation des brebis Chios n'est pas contrôlé par un gène à effet majeur.

Pour la conduite de l'élevage ovin grec, la mise en évidence de très fortes variations saisonnières du taux d'ovulation, en particulier chez la brebis Chios, peut avoir des conséquences importantes. En effet, la période de reproduction est habituellement organisée avec une lutte de printemps, période à laquelle l'activité sexuelle, en particulier le taux d'ovulation, est à son plus bas niveau de l'année. Cette faible activité au printemps pourrait expliquer la grande dispersion des fécondations enregistrée après «l'effet bélier» qui suit l'introduction des mâles (Michailidis, 1986). La comparaison d'une lutte d'automne, permettant de tirer partie de la pleine activité sexuelle des brebis à cette saison, avec une lutte traditionnelle de printemps, serait intéressante à conduire afin de connaître la différence de producti- 
vité numérique des brebis à ces deux périodes.

\section{REMERCIEMENTS}

Les auteurs remercient pour leur aide $T$ Alifakiotis, I Michailidis et $V$ Vergos ainsi que l'ensemble du personnel des stations de Khalkidiki et de Serres qui ont participé à cette expérimentation.

\section{RÉFÉRENCES}

Alifakiotis T (1986) Increasing ovulation rate and lambing percentage by active immunization against androstenedione in dairy sheep breeds. Theriogenology 25, 681-688

Bindon BM (1984) Reproductive biology of the Boroola Merino sheep. Aust J Biol Sci 37, 163-189

Chemineau P, Daveau A, Maurice F, Delgadillo $J$ A (1992) Seasonality of oestrus and ovulation is not modified by submitting female Alpine goats to a tropical photoperiod. Small Rum Res 8, 299-312

Cornu C, Cognié Y (1985) The utilization of Romanov sheep in a system of integrated husbandry. In: Genetics of Reproduction in Sheep (RB Land, DW Robinson eds) Butterworths, Londres, 383-389

Dagnélie P (1970) Théorie et méthodes statistiques. Presses Agronomiques de Gembloux, Gembloux, Belgique, Vol 2, $451 \mathrm{p}$

Hafez ESE (1952) Studies on the breeding season and reproduction of the ewe. J Agric SCi Camb 42, 189-265

Hatjiminaoglou J, Zervas N, Boyazoglu J (1985) Sheep and goats in Greece. Publ Fac Agric Univ Thessaloniki Tessalonique, Grèce, $29 \mathrm{p}$

Khaldi G (1984) Variations saisonnières de l'activité ovarienne, du comportement d'œstrus et de la durée de l'ancestrus post-partum des femelles ovines de race barbarine : influences du niveau alimentaire et de la présence du mâle. Thèse Doct Etat ès Sci Univ Sci Tech Languedoc L, $168 \mathrm{p}$

Lahlou-Kassi A, Marie M (1985) Sexual and ovarian function of the D'Man ewe. In: Genetics of reproduction in sheep (RB Land and
DW Robinson Eds) Butterworths, Londres, 245-260

Lysandrides P (1987) The Chios sheep in Cyprus. In: Small ruminants in the near east, vol 2, FAO Anim Prod and Health paper $\mathrm{N}^{\circ} 55$, 18-22

Mahieu $M$, Jégo $Y$, Driancourt MA, Chemineau $P$ (1989) Reproductive performances of Creole and Black-Belly ewes in the West Indies. A new major gene controlling ovulation rate? Anim Reprod Sci 19, 235-243

Mauléon P, Dauzier L (1965) Variations de durée de l'ancestrus de lactation chez les brebis de race lle-de-France. Ann Biol Anim Biochim Biophys 5,131-143

Michailidis IG (1986) Parameters of reproduction in Chios and Kimis ewes. PhD Thesis, University of Thessaloniki, $270 \mathrm{p}$

Perret G (1986) Races ovines. ITOVIC-SPEOC, Paris, $441 p$

Quirke JF, Stabenfeldt GH, Bradford GE (1988) Year and season effects on oestrus and ovarian activity in ewes of different breeds and crosses. Anim Reprod Sci 16, 39-52

Terqui M, Thimonier J (1974) Nouvelle méthode radioimmunologique rapide pour l'estimation du niveau de progestérone plasmatique. Application pour le diagnostic précoce de la gestation chez la brebis et la chèvre. CR Heb Séance Acad Sci Paris, D, 279, 1109-1112

Thimonier J (1978) L'activité ovarienne chez les bovins : moyens d'étude et facteurs de variations. Ann Méd Vét 122, 81-92

Thimonier J (1989) Contrôle photopériodique de l'activité ovulatoire chez la brebis lle-deFrance. Existence de rythmes endogènes. Thèse Doctorat Université de Tours, $112 p$

Thimonier J, Mauléon P (1969) Variations saisonnières du comportement d'œstrus et des activités ovarienne et hypophysaire chez les ovins. Ann Biol Anim Biochim Biophys 9, 233-25

Vosniakou AG, Doney J M, Tsakalof P (1989) A note on the seasonal oestrous period in three breeds of greek dairy sheep. Anim Prod 49, 147-15

Young LD, Dickerson GE, Fogarty NM (1985) Evaluation and utilization of Finn sheep. In: Genetics of reproduction in sheep (RB Land and DW Robinson eds) Butterworths, Londres, 25-38 